Table S3.

\begin{tabular}{lll}
\hline & SIO & NIO \\
\hline Mutation model & F84 & F84 \\
Transition/transversion ratio & 17.243159 & 11.329992 \\
Site rate modifier (4 groups) & 0.3225991 .7449424 .5336869 .388189 & 0.3232631 .7343734 .4957969 .299330 \\
Probabilities of site rates & 0.6029570 .3575820 .0389220 .000540 & 0.6003900 .3596990 .0393630 .000548 \\
Prior distribution for mutation-scaled population size & Uniform with range 0.0 to 1.5 & Uniform with range 0.0 to 1.0 \\
Prior distribution for mutation-scaled migration rates & Uniform with range 0.0 to 5000 & Uniform with range 0.0 to 2000 \\
Proposal distribution for parameters and geneaologies & Slice sampling & Slice sampling \\
Increment between samples & 200 & 200 \\
Samples per replicate & $5 ' 000$ & $5 ' 000$ \\
Burn-ins per replicate & $100 ' 000$ & 100 '000 \\
Replicates & 32 & 32 \\
\hline
\end{tabular}




\section{Table S4.}

(a) Northern Indian Ocean

\begin{tabular}{|c|c|c|c|c|c|c|c|c|}
\hline & UAE & Oman & Maldives & Thailand & Aceh & XmasIs & Seribu & Krakatau \\
\hline Oman & 0.010 & & & & & & & \\
\hline Maldives & 0.551 & 0.505 & & & & & & \\
\hline Thailand & 0.776 & 0.757 & 0.157 & & & & & \\
\hline Aceh & 0.741 & 0.716 & 0.077 & 0.039 & & & & \\
\hline Christmas Island & 0.893 & 0.905 & 0.433 & 0.372 & 0.454 & & & \\
\hline Seribu & 0.777 & 0.756 & 0.129 & -0.037 & 0.025 & 0.401 & & \\
\hline Karimunjawa & 0.796 & 0.777 & 0.068 & 0.075 & -0.010 & 0.524 & -0.002 & 0.021 \\
\hline
\end{tabular}

Values in bold were significant after Bonferroni correction: $p<0.00139$.

(b) Southern Indian Ocean

\begin{tabular}{|c|c|c|c|c|c|c|c|c|c|c|}
\hline & UAE & Oman & Chagos & CocosIs & SthAfrica & Kenya & Mauritius & Reunion & Mayotte & NthMadagascar \\
\hline Oman & 0.000 & & & & & & & & & \\
\hline Chagos & 0.070 & 0.149 & & & & & & & & \\
\hline CocosIs & 0.623 & 0.508 & 0.249 & & & & & & & \\
\hline SthAfrica & 0.032 & 0.086 & -0.013 & 0.174 & & & & & & \\
\hline Kenya & -0.059 & 0.091 & -0.017 & 0.144 & 0.013 & & & & & \\
\hline Mauritius & 0.399 & 0.263 & 0.069 & 0.545 & -0.012 & 0.079 & & & & \\
\hline Mayotte & -0.108 & 0.054 & 0.005 & 0.167 & 0.021 & -0.002 & 0.137 & -0.015 & & \\
\hline NthMadagascar & -0.143 & 0.016 & -0.020 & 0.177 & 0.029 & -0.023 & 0.117 & 0.006 & -0.037 & \\
\hline SthMadagascar & -0.042 & -0.667 & 0.165 & 0.444 & 0.148 & 0.128 & 0.258 & 0.194 & 0.122 & 0.049 \\
\hline
\end{tabular}

Values in bold were significant after Bonferroni correction: $p<0.0009$. 